International Journal of Modern Physics B,

(C) World Scientific Publishing Company

\title{
MODEL CALCULATION OF ELECTRON-PHONON COUPLINGS IN A DIMER WITH A NON-DEGENERATE ORBITAL
}

\author{
M. ACQUARONE \\ C.N.R.-G.N.S.M., Unita' I.N.F.M. di Parma, Dipartimento di Fisica \\ Universita' di Parma, Parma, I-43100, Italy \\ and \\ C. NOCE \\ Unità I.N.F.M., Dipartimento di Scienze Fisiche "E.R. Caianiello" \\ Università di Salerno, Baronissi, I-84081, Italy
}

Received ( )

Revised ( )

\begin{abstract}
We evaluate all the electron-phonon couplings derived from the one-body and two-body electronic interactions, in both the adiabatic and extreme non-adiabatic limit, for a dimer with a non-degenerate orbital built from atomic wave functions of Gaussian shape. We find largely different values of the coupling parameters in the two limits, as well as different expressions of the corresponding terms in the Hamiltonian. Depending on the distance between the dimer ions, some of the two-body couplings are comparable, or even larger than the one-body ones.
\end{abstract}

\section{Introduction}

The models containing interactions of electrons and phonons have been the object of intense study in the recent years, mainly due to the discovery of high $T_{c}$ superconductors $(H T S)$ and colossal magnetoresistance manganites $(C M R)$. When trying to apply the theoretical results to the real materials, however, one is confronted with the difficulty that a large degree of arbitrariness is present, stemming from two origins. First, different forms of interactions have been introduced, essentially on an $a d h o c$ basis. Second, the value of the interaction parameters are estimated from the experimental data, which, however, can be interpreted only after a definite theoretical model, i.e. a set of electron-phonon interactions, has been assumed to be relevant for the material under measurement. Therefore, besides unavoidable quantitative approximations in fitting the data, also qualitative uncertainties are introduced a priori in the analysis. The purpose of the present work is to provide, in a simple but not trivial model, both rigorous prescriptions about the admissible electron-phonon interaction terms, and the quantitative evaluation of the interaction strength parameters. The model we consider is a dimer where the electrons occupy a non-degenerate orbital described by Wannier functions built from atomic orbitals of Gaussian shape. All the one- and two-body electronic parameters in that 
model have been analytically evaluated in Ref.1. The electron-phonon interactions will be evaluated following the general definitions introduced in Refs. 2 and 3.

The structure of the paper is as follows: in Sec. 2 we describe the model and the general procedure of evaluation; Secs. 3 and 4 are devoted to the analytical evaluation of the coupling parameters in the non-adiabatic, and adiabatic limits, respectively, also defining the correct form of the corresponding terms in the Hamiltonian; Sec.5 discusses the numerical evaluation of the coupling parameters; Sec.6 is devoted to the conclusions. The Appendices contain details of the analytical calculations.

\section{The Model}

In a general electron-phonon Hamiltonian $H=H_{e l}+H_{p h}+H_{e l-p h}$ the interacting term $H_{e l-p h}$ originates from the development of the electronic part $H_{e l}$ to first order in the phonon-induced displacement of the ion positions. In our analysis we shall consider the terms due to the development of both the one-body and two-body contributions to the electronic Hamiltonian, which, in standard notation 1 , reads:

$$
\begin{aligned}
H_{e l}= & \epsilon \sum_{\sigma}\left(n_{1 \sigma}+n_{2 \sigma}\right)+\sum_{\sigma}\left[t+X\left(n_{1-\sigma}+n_{2-\sigma}\right)\right]\left(c_{1 \sigma}^{\dagger} c_{2 \sigma}+H . c .\right)+U\left(n_{1 \uparrow} n_{1 \downarrow}+n_{2 \uparrow} n_{2 \downarrow}\right) \\
& +V n_{1} n_{2}-2 J_{z} S_{1}^{z} S_{2}^{z}-J_{x y}\left(S_{1}^{+} S_{2}^{-}+H . c .\right)+P\left(c_{1 \uparrow}^{\dagger} c_{1 \downarrow}^{\dagger} c_{2 \downarrow} c_{2 \uparrow}+H . c .\right) .
\end{aligned}
$$

The electrons are assumed to occupy a non-degenerate orbital described by Wannier functions built from atomic orbitals of Gaussias shape.

\subsection{Undisplaced Wannier functions}

The ions occupy the undeformed positions $\boldsymbol{R}_{1}^{0}=(-a / 2,0,0)$ and $\boldsymbol{R}_{2}^{0}=(a / 2,0,0)$. The unit vector $\boldsymbol{e}_{12}=\left(\boldsymbol{R}_{2}^{0}-\boldsymbol{R}_{1}^{0}\right) / a=(1,0,0)$ points from ion 1 towards ion 2 ; if not specified otherwise, $\boldsymbol{e}=\boldsymbol{e}_{12}$. The position of the electron is $\boldsymbol{r}=(x, y, z)$. We associate to each site $i=1,2$ a Gaussian atomic-like orbital $\phi_{i}\left(\boldsymbol{r}-\boldsymbol{R}_{i}\right)$. By defining $N \equiv(2 / \pi)^{3 / 4} \Gamma^{3 / 2}$, so that $\left\langle\phi_{i} \mid \phi_{i}\right\rangle=1$, they read:

$$
\begin{aligned}
& \phi_{1}\left(\boldsymbol{r}-\boldsymbol{R}_{1}^{0}\right)=N \exp \left\{-\Gamma^{2}\left[(x+a / 2)^{2}+y^{2}+z^{2}\right]\right\}, \\
& \phi_{2}\left(\boldsymbol{r}-\boldsymbol{R}_{2}^{0}\right)=N \exp \left\{-\Gamma^{2}\left[(x-a / 2)^{2}+y^{2}+z^{2}\right]\right\} .
\end{aligned}
$$

Their overlap $S_{0} \equiv\left\langle\phi_{1} \mid \phi_{2}\right\rangle=\exp \left(-\Gamma^{2} a^{2} / 2\right)$ is non-vanishing. Then the Wannier functions $\Psi_{1}, \Psi_{2}$ can be written as:

$$
\begin{aligned}
& \Psi_{1}\left(\boldsymbol{r}-\boldsymbol{R}_{1}^{0}, \boldsymbol{r}-\boldsymbol{R}_{2}^{0}\right)=A\left(S_{0}\right) \phi_{1}\left(\boldsymbol{r}-\boldsymbol{R}_{1}^{0}\right)+B\left(S_{0}\right) \phi_{2}\left(\boldsymbol{r}-\boldsymbol{R}_{2}^{0}\right), \\
& \Psi_{2}\left(\boldsymbol{r}-\boldsymbol{R}_{1}^{0}, \boldsymbol{r}-\boldsymbol{R}_{2}^{0}\right)=B\left(S_{0}\right) \phi_{1}\left(\boldsymbol{r}-\boldsymbol{R}_{1}^{0}\right)+A\left(S_{0}\right) \phi_{2}\left(\boldsymbol{r}-\boldsymbol{R}_{2}^{0}\right) .
\end{aligned}
$$

The requirement $\left\langle\Psi_{i} \mid \Psi_{j}\right\rangle=\delta_{i j}$ yields the mixing coefficients $A, B$ as:

$$
A\left(S_{0}\right) \equiv \frac{1}{2}\left[\frac{1}{\sqrt{1+S_{0}}}+\frac{1}{\sqrt{1-S_{0}}}\right], \quad B\left(S_{0}\right) \equiv \frac{1}{2}\left[\frac{1}{\sqrt{1+S_{0}}}-\frac{1}{\sqrt{1-S_{0}}}\right] .
$$


In the limit $S_{0} \rightarrow 0$, one has $\Psi_{i} \rightarrow \phi_{i}(i=1,2)$, so that one can connect unambiguously each Wannier function to a well defined site.

\subsection{Displaced Wannier functions}

We shall consider only deformations $\boldsymbol{u}_{1}=u_{1} \boldsymbol{e}_{12}$ and $\boldsymbol{u}_{2}=u_{2} \boldsymbol{e}_{12}$ altering the length of the dimer, so that only their $x$-components are non vanishing. Their signs are defined, on both sites, with respect to the same unit vector $\boldsymbol{e}_{12}$, so as to have a single reference system. Symmetry requirements impose $\boldsymbol{u}_{1}=-\boldsymbol{u}_{2}$ so that the relative displacement $u \equiv u_{2}-u_{1}=-2 u_{1}=2 u_{2}$. Even though we shall never introduce the exact time dependency in the following calculation, it is important to keep in mind that the displacements vary periodically in time with the frequency $\Omega$ of the phonon. The instantaneous positions of the ions are therefore defined as

$$
\boldsymbol{R}_{1}=\boldsymbol{R}_{1}^{0}+\boldsymbol{u}_{1}=\left(-a / 2+u_{1}, 0,0\right), \quad \boldsymbol{R}_{2}=\boldsymbol{R}_{2}^{0}+\boldsymbol{u}_{2}=\left(a / 2+u_{2}, 0,0\right) .
$$

In the adiabatic limit, the orbitals on each site are assumed to adjust instantaneously to the displaced positions of the ions. Then, if we define

$$
\begin{aligned}
& \phi_{1}\left[\boldsymbol{r}-\left(\boldsymbol{R}_{1}^{0}+\boldsymbol{u}_{1}\right)\right]=N \exp \left\{-\Gamma^{2}\left[\left(x+a / 2-u_{1}\right)^{2}+y^{2}+z^{2}\right]\right\}, \\
& \phi_{2}\left[\boldsymbol{r}-\left(\boldsymbol{R}_{2}^{0}+\boldsymbol{u}_{2}\right)\right]=N \exp \left\{-\Gamma^{2}\left[\left(x-a / 2-u_{2}\right)^{2}+y^{2}+z^{2}\right]\right\},
\end{aligned}
$$

the Wannier functions $\Psi_{i}(i=1,2)$ are written as:

$$
\begin{gathered}
\Psi_{1}\left(\boldsymbol{R}_{1}^{0}, \boldsymbol{R}_{2}^{0}, u_{1}, u_{2}\right)=A\left(u_{1}, u_{2}\right) \phi_{1}\left(\boldsymbol{r}-\boldsymbol{R}_{1}^{0}-\boldsymbol{u}_{1}\right)+B\left(u_{1}, u_{2}\right) \phi_{2}\left(\boldsymbol{r}-\boldsymbol{R}_{2}^{0}-\boldsymbol{u}_{2}\right), \\
\Psi_{2}\left(\boldsymbol{R}_{1}^{0}, \boldsymbol{R}_{2}^{0}, u_{1}, u_{2}\right)=B\left(u_{1}, u_{2}\right) \phi_{1}\left(\boldsymbol{r}-\boldsymbol{R}_{1}^{0}-\boldsymbol{u}_{1}\right)+A\left(u_{1}, u_{2}\right) \phi_{2}\left(\boldsymbol{r}-\boldsymbol{R}_{2}^{0}-\boldsymbol{u}_{2}\right) .
\end{gathered}
$$

In principle we have four coefficients $A\left(u_{1}, u_{2}\right), B\left(u_{1}, u_{2}\right)$ to determine. We make the reasonable guess that, as for $u_{i} \rightarrow 0$ the $A$ 's ( and the $B$ 's) have the same shape on both sites, then they keep the same shape also for $u_{i} \neq 0$. We shall define $A_{0}, B_{0}, S_{0}$ as $\lim _{u_{1}, u_{2} \rightarrow 0} A\left(u_{1}, u_{2}\right)$ etc. As $\left\langle\phi_{1}\left(u_{1}\right) \mid \phi_{1}\left(u_{1}\right)\right\rangle=\left\langle\phi_{2}\left(u_{2}\right) \mid \phi_{2}\left(u_{2}\right)\right\rangle=1$ still holds, by defining $S \equiv \exp \left\{-\left(\Gamma^{2} / 2\right)\left[a-\left(u_{2}-u_{1}\right)\right]^{2}\right\}$ the condition $\left\langle\Psi_{i}\left(u_{1}, u_{2}\right) \mid \Psi_{j}\left(u_{1}, u_{2}\right)\right\rangle=$ $\delta_{i j}$ now yields:

$$
A(S) \equiv \frac{1}{2}\left[\frac{1}{\sqrt{1+S}}+\frac{1}{\sqrt{1-S}}\right], \quad B(S) \equiv \frac{1}{2}\left[\frac{1}{\sqrt{1+S}}-\frac{1}{\sqrt{1-S}}\right] .
$$

Namely, $A(S)$ and $B(S)$ depend on $S(u)$ as $A_{0}$ and $B_{0}$ depend on $S_{0}$.

\subsection{The electronic parameters}

Given the Wannier functions, all the electronic parameters can be evaluated: this was done in Ref.1. To make clear our method of calculation, it is convenient to explicitate the one-body electronic interactions, corresponding to the local energy $\epsilon$ and to the hopping amplitude $t$. 


$$
\begin{aligned}
\epsilon_{i} & =\int \Psi_{i}^{*}\left(\boldsymbol{r}, \boldsymbol{R}_{1}, \boldsymbol{R}_{2}\right)\left[-\frac{\hbar^{2}}{2 m} \nabla^{2}+V_{1}\left(\boldsymbol{r}-\boldsymbol{R}_{1}\right)+V_{2}\left(\boldsymbol{r}-\boldsymbol{R}_{2}\right)\right] \Psi_{i}\left(\boldsymbol{r}, \boldsymbol{R}_{1}, \boldsymbol{R}_{2}\right) d^{3} \boldsymbol{r}, \\
t & =\int \Psi_{1}^{*}\left(\boldsymbol{r}, \boldsymbol{R}_{1}, \boldsymbol{R}_{2}\right)\left[-\frac{\hbar^{2}}{2 m} \nabla^{2}+V_{1}\left(\boldsymbol{r}-\boldsymbol{R}_{1}\right)+V_{2}\left(\boldsymbol{r}-\boldsymbol{R}_{2}\right)\right] \Psi_{2}\left(\boldsymbol{r}, \boldsymbol{R}_{1}, \boldsymbol{R}_{2}\right) d^{3} \boldsymbol{r} .
\end{aligned}
$$

In the formulas above, the potentials originating from the ion cores at the displaced positions $\boldsymbol{R}_{1}$ and $\boldsymbol{R}_{2}$ are:

$$
\begin{aligned}
& V_{1} \equiv V\left(\boldsymbol{r}-\boldsymbol{R}_{1}\right)=-e^{2} Z\left[\left(x+\frac{a}{2}-u_{1}\right)^{2}+y^{2}+z^{2}\right]^{-1 / 2}, \\
& V_{2} \equiv V\left(\boldsymbol{r}-\boldsymbol{R}_{2}\right)=-e^{2} Z\left[\left(x-\frac{a}{2}-u_{2}\right)^{2}+y^{2}+z^{2}\right]^{-1 / 2},
\end{aligned}
$$

where $-e$ is the electron charge, and $+Z e$ is the charge of the ion core.

The local energy $\epsilon$ (actually site-independent) can be decomposed into three terms, respectively corresponding to the contributions from the Laplacian kinetic operator $\left(\epsilon_{\nabla}\right)$ and from each one of the ionic potentials $\left(\epsilon_{V_{1}}, \epsilon_{V_{2}}\right)$. The modulation of the term where both charge distribution and potential refer to the same ion gives rise to an Holstein-type coupling. The other term, where the charge around one ions feels the displaced potential of the other ion, we shall call "crystal-field" coupling. In the literature 3 this latter term is usually assumed to be negligible but we shall see that actually is of the same order as the other ones, and can even become the dominant one.

The modulation of the hopping amplitude $t$ gives rise to the so-called SuSchrieffer-Heeger $(S S H)$ interactions. Similarly to $\epsilon$, it can be decomposed into kinetic $\left(t_{\nabla}\right)$ and potential $\left(t_{V_{1}, V_{2}}\right)$ contributions.

We need to distinguish between the adiabatic (labelled $a d$ ) and the non-adiabatic (labelled $n a$ ) limit in evaluating the electron-phonon interactions, because the integrals have different kernels in the two cases. Indeed, in the adiabatic limit, when $\hbar \Omega<t$, the displacements affect both the potentials and the electronic Wannier functions, expressing the requisite that the electronic charge distribution adjusts itself instantaneously at the position of the ions.

We shall schematize the opposite situation $\hbar \Omega>t$, where the electrons are slower than the ions, as realized by the electronic charge distribution staying centred around the undisplaced ion position, while the potentials are centred on the displaced ions. We shall call this the extreme anti-adiabatic limit. Not only the strength of the interactions, but also the form of the terms contributing to the Hamiltonian will turn out to differ in the two cases.

The anti-adiabatic limit will be treated first, as the notation is easier to establish in that case. 


\section{Couplings in the non-adiabatic limit}

If the Wannier functions keep being centred around the undisplaced ionic positions, neither $\epsilon_{\nabla}$ nor $t_{\nabla}$ does change, therefore no electron-phonon coupling originates from them. The couplings derived from the two-body interactions are also identically vanishing in this limit, because they involve the Wannier functions and the inter-electronic Coulomb potential which are both insensitive to the displacements of the ions.

The only non-vanishing types of electron-phonon non-adiabatic couplings are those arising from the variation of the potential contributions to $\epsilon$ and $t$.

\subsection{The Holstein-type coupling $g_{0}$}

The Holstein-type coupling $g_{0}$ is usually introduced as the site-independent amplitude of a term in the Hamiltonian connecting the local charge with the local deformation:

$$
g_{0} \sum_{\sigma}\left(n_{1 \sigma} u_{1}+n_{2 \sigma} u_{2}\right)
$$

This interaction originates from the perturbation of the on-site atomic energy $\epsilon_{0}$ due to the ion motion in the non-adiabatic limit, namely:

$$
\begin{gathered}
\left\langle\Psi_{i}\left(\boldsymbol{R}_{1}^{0}, \boldsymbol{R}_{2}^{0}\right)\left|V_{i}\left(\boldsymbol{R}_{i}\right)\right| \Psi_{i}\left(\boldsymbol{R}_{1}^{0}, \boldsymbol{R}_{2}^{0}\right)\right\rangle-\left\langle\Psi_{i}\left(\boldsymbol{R}_{1}^{0}, \boldsymbol{R}_{2}^{0}\right)\left|V_{i}\left(\boldsymbol{R}_{i}^{0}\right)\right| \Psi_{i}\left(\boldsymbol{R}_{1}^{0}, \boldsymbol{R}_{2}^{0}\right)\right\rangle \\
\equiv g_{0}^{(i)} n_{i} u_{i}+\mathcal{O}\left(u_{i}^{2}\right), \quad(i=1,2)
\end{gathered}
$$

Our $g_{0}^{(i)}$ is therefore different from the interaction defined by Holstein 6 because he considered the interaction as due to the variation of the internuclear distance between the two components of each dimer attached at the nodes of a frozen chain. We use the "Holstein" label because this interaction has the same form as the traditional Holstein term. We also allow for a possible site-dependency of $g_{0}^{(i)}$, to show later that this is not the case.

For the ion at $\boldsymbol{R}_{1}$ one writes $g_{0}^{(1)}$ as:

$$
g_{0}^{(1)} \equiv\left(\lim _{u_{1} \rightarrow 0} \frac{\partial \epsilon_{V_{1}}^{(1)}}{\partial u_{1}}\right)=\left(\lim _{u_{1} \rightarrow 0} \frac{\partial}{\partial u_{1}}\right) \int_{-\infty}^{\infty} \Psi_{1}\left(x, y, z, \boldsymbol{R}_{1}^{0}, \boldsymbol{R}_{2}^{0}\right)^{2} V_{1}\left(u_{1}\right) d x d y d z
$$

In the integral

$$
\begin{gathered}
\int_{-\infty}^{\infty} \Psi_{1}\left(x, y, z, \boldsymbol{R}_{1}^{0}, \boldsymbol{R}_{2}^{0}\right)^{2} V_{1}\left(u_{1}\right) d x d y d z= \\
\int_{-\infty}^{\infty}\left\{A \phi_{1}\left[\left(x+\frac{a}{2}\right), y, z\right]+B \phi_{2}\left[\left(x-\frac{a}{2}\right), y, z\right]\right\}^{2} V_{1}\left[\left(x+\frac{a}{2}-u_{1}\right), y, z\right] d x d y d z
\end{gathered}
$$

let us change variable form $x$ to $p=x+a / 2-u_{1}$, to shift the origin of $x$ coordinate onto the displaced ion, without changing the shape of the Wannier functions. The 
reason is to avoid the derivation with respect to $u_{1}$ of $V_{1}\left(u_{1}\right)$ which is discontinuous in the integration range. The above expression changes into:

$$
\int_{-\infty}^{\infty}\left\{A \phi_{1}\left[\left(p+u_{1}\right), y, z\right]+B \phi_{2}\left[\left(p-a+u_{1}\right), y, z\right]\right\}^{2} V_{1}[(p, y, z)] d x d y d z .
$$

In this form the kernel has a continuous derivative with respect to $u_{1}$ everywhere. Notice also that $A$ and $B$ still do not depend on $u_{1}$. To derive with respect to $u_{1}$ Eq.16, we use $u_{2}=-u_{1}$ and notice that:

$\lim _{u_{1} \rightarrow 0} \frac{\partial \phi_{1}\left(p+u_{1}, y, z\right)}{\partial u_{1}}=\frac{\partial \phi_{1}(x, y, z)}{\partial x}, \quad \lim _{u_{2} \rightarrow 0} \frac{\partial \phi_{2}\left(p-a-u_{2}, y, z\right)}{\partial u_{2}}=-\frac{\partial \phi_{2}(x, y, z)}{\partial x}$,

yielding:

$$
\begin{gathered}
\lim _{u_{1} \rightarrow 0} \frac{\partial}{u_{1}}\left\{A \phi_{1}\left[\left(p+u_{1}\right), y, z\right]+B \phi_{2}\left[\left(p-a+u_{1}\right), y, z\right]\right\}^{2} \\
=2 \Psi_{1}\left[A \frac{\partial \phi_{1}}{\partial x}+B \frac{\partial \phi_{2}}{\partial x}\right]=2 \Psi_{1} \frac{\partial \Psi_{1}}{\partial x} .
\end{gathered}
$$

Therefore we can write:

$$
g_{0}^{(1)}=2 \int_{-\infty}^{\infty} \Psi_{1} \frac{\partial \Psi_{1}}{\partial x} V_{1} d x d y d z
$$

By substituting $\Psi_{1}$ and its derivative into Eq.19 and by noticing that $\left\langle\phi_{1}\left|(x+a / 2) V_{1}\right| \phi_{1}\right\rangle=$ 0 , as the kernel is odd in $x+a / 2$, we obtain:

$$
g_{0}^{(1)}=-4 \Gamma^{2} \int_{-\infty}^{\infty}\left(B^{2} \phi_{2}^{2}+2 A B \phi_{1} \phi_{2}\right) x V_{1} d x d y d z+2 \Gamma^{2} a B^{2} \int_{-\infty}^{\infty} \phi_{2}^{2} V_{1} d x d y d z .
$$

The integrals in Eq.20 are listed in Appendix 2. The final result is:

$$
g_{0}^{(1)}=\frac{2 \Gamma \sqrt{2 / \pi}}{a}\left\{B^{2}\left[F_{0}\left(2 a^{2} \Gamma^{2}\right)-S^{4}\right]+4 A B S\left[F_{0}\left(\frac{a^{2} \Gamma^{2}}{2}\right)-S\right]\right\} .
$$

That $g_{0}^{(i)}$ is site-independent can be proved by considering that, for equal charges and displacement amplitudes, the energies $E^{(i)}=g_{0}^{(i)} n_{i} \mathbf{u}_{i} \bullet \mathbf{e}_{i j}(i, j=1,2)$ on both sites must coincide, i.e.

$$
g_{0}^{(1)} \mathbf{u}_{1} \bullet \mathbf{e}_{12}=g_{0}^{(2)} \mathbf{u}_{2} \bullet \mathbf{e}_{21}=-g_{0}^{(2)} \mathbf{u}_{2} \bullet \mathbf{e}_{12}
$$

Now symmetry requires $u_{1}=-u_{2}$ from which $g_{0}^{(2)}=g_{0}^{(1)} \equiv g_{0}$ follows.

\subsection{The crystal-field coupling}

This term expresses the change in the energy of interaction between the charge on site $i$ and the potential centred on the site $j$, that is:

$$
\left\langle\Psi_{i}^{0}\left|V_{j}\left(\boldsymbol{R}_{j}^{0}+u_{j}\right)\right| \Psi_{i}^{0}\right\rangle-\left\langle\Psi_{i}^{0}\left|V_{j}\left(\boldsymbol{R}_{j}^{0}\right)\right| \Psi_{i}^{0}\right\rangle \equiv g_{c f}^{(i)} n_{i} u_{j}+\mathcal{O}\left(\sqcap_{\mid}^{\in}\right)
$$


In the literature, there is some confusion on the form of this term, so we shall discuss it in some details, following the line of reasoning used to prove that $g_{0}^{(1)}=$ $g_{0}^{(2)}$. Consider site 1 , with charge $n_{1}$. Its energy, after a displacement $\mathbf{u}_{2}$, changes by an amount $E^{(1)}=g_{c f}^{(1)} n_{1} \mathbf{u}_{2} \bullet \mathbf{e}_{12}$. This can be considered as the quantity measured by an observer sitting on ion 1 and watching the ion 2 moved by $\mathbf{u}_{2}$ . The equivalent measurement done by an observer on ion 2 watching the ion 1 displaced by $\mathbf{u}_{1}$, yields $E^{(2)}=g_{c f}^{(2)} n_{2} \mathbf{u}_{1} \bullet \mathbf{e}_{21}$. Notice that each observer uses its own unit vector, pointing from the observer's ion towards the other, displaced ion. Formally, $E^{(2)}$ can be obtained by performing a site label permutation on $E^{(1)}$. An external observer would find it more convenient to refer both quantities to the same reference system, $\mathbf{e}_{12}$ say, yielding $E^{(2)}=-g_{c f}^{(2)} n_{2} \mathbf{u}_{1} \bullet \mathbf{e}_{12}$. Assuming equal charge density and displacement amplitude in the two observations, one must have $E^{(1)}=E^{(2)}$. It follows, dropping the charge densities:

$$
g_{c f}^{(1)} \mathbf{u}_{2} \bullet \mathbf{e}_{12}=-g_{c f}^{(2)} \mathbf{u}_{1} \bullet \mathbf{e}_{12} \Longrightarrow g_{c f}^{(1)} u_{1}=-g_{c f}^{(2)} u_{2} \Longrightarrow g_{c f}^{(1)}=g_{c f}^{(2)}=g_{c f},
$$

because the only displacements allowed by symmetry are such that $u_{1}=-u_{2}$. Therefore for the dimer as a whole one writes this term as

$$
g_{c f} \sum_{\sigma}\left(n_{1 \sigma} u_{2}+n_{2 \sigma} u_{1}\right) .
$$

Passing now to the explicit evaluation for site 1 we have:

$$
g_{c f}^{(1)}=\lim _{u_{2} \rightarrow 0} \frac{\partial}{\partial u_{2}} \int_{-\infty}^{\infty} \Psi_{1}\left(\boldsymbol{R}_{1}^{0}, \boldsymbol{R}_{2}^{0}, x, y, z\right)^{2} V_{2}\left(u_{2}\right) d x d y d z .
$$

We change variables from $x$ to $p=x / a / 2-u_{2}$ and proceed in strict analogy to the evaluation of $g_{0}^{(1)}$, arriving at:

$$
g_{c f}^{(1)}=2 \int_{-\infty}^{\infty} \Psi_{1} \frac{\partial \Psi_{1}}{\partial x} V_{2} d x d y d z .
$$

By explicitating $\Psi_{1}$ and its derivative we obtain:

$$
\begin{aligned}
g_{c f}^{(1)}=- & 4 \Gamma^{2} \int_{-\infty}^{\infty}\left(A^{2} \phi_{1}^{2}+B^{2} \phi_{2}^{2}+2 A B \phi_{1} \phi_{2}\right) x V_{2} d x d y d z \\
& -2 \Gamma^{2} a \int_{-\infty}^{\infty}\left(A^{2} \phi_{1}^{2}-B^{2} \phi_{2}^{2}\right) V_{2} d x d y d z .
\end{aligned}
$$

¿From the matrix elements evaluated in Appendix 2 we obtain:

$$
g_{c f}^{(1)}=-2 A\left(\frac{\Gamma}{a}\right) \sqrt{\frac{2}{\pi}}\left[A F_{0}\left(2 a^{2} \Gamma^{2}\right)+4 B S F_{0}\left(a^{2} \Gamma^{2} / 2\right)-4 B S^{2}-A S^{4}\right] .
$$




\subsection{The Su-Schrieffer-Heeger coupling}

This term is a non-diagonal inter-site coupling, due to the modulation of the hopping amplitude $t$. As the Wannier functions keep being centred on the static lattice positions, the difference in kinetic energy caused by the displacements is due only to the contribution to $t$ from $V_{1}$ and $V_{2}$ :

$$
\begin{gathered}
\int \Psi_{1}\left(\boldsymbol{r}-\boldsymbol{R}_{1}^{0}\right)\left[V_{1}\left(\boldsymbol{r}-\boldsymbol{R}_{1}^{0}-\boldsymbol{u}_{1}\right)+V_{2}\left(\boldsymbol{r}-\boldsymbol{R}_{2}^{0}-\boldsymbol{u}_{2}\right)\right] \Psi_{2}\left(\boldsymbol{r}-\boldsymbol{R}_{2}^{0}\right) d^{3} \boldsymbol{r} \\
-\int \Psi_{1}\left(\boldsymbol{r}-\boldsymbol{R}_{1}^{0}\right)\left[V_{1}\left(\boldsymbol{r}-\boldsymbol{R}_{1}^{0}\right)+V_{2}\left(\boldsymbol{r}-\boldsymbol{R}_{2}^{0}\right)\right] \Psi_{2}\left(\boldsymbol{r}-\boldsymbol{R}_{2}^{0}\right) d^{3} \boldsymbol{r} \\
\equiv \gamma_{12} \sum_{\sigma}\left(c_{1 \sigma}^{\dagger} c_{2 \sigma}+c_{2 \sigma}^{\dagger} c_{1 \sigma}\right)\left(u_{2}-u_{1}\right)+\mathcal{O}\left[\left(u_{2}-u_{1}\right)^{2}\right] .
\end{gathered}
$$

Notice that, to preserve the invariance of the Hamiltonian under site permutation, the $S S H$ coupling has to be odd under the same operation: $\gamma_{12}=-\gamma_{21}$ (see e.g Refs. 2,3).

Let us distinguish the two contributions as $t_{V_{1}}$ and $t_{V_{2}}$, defined as:

$$
\begin{aligned}
& t_{V_{1}}=\int_{-\infty}^{\infty} \frac{\Psi_{1}(u=0) \Psi_{2}(u=0)}{\sqrt{\left(x+a / 2-u_{1}\right)^{2}+y^{2}+z^{2}}} d x d y d z, \\
& t_{V_{2}}=\int_{-\infty}^{\infty} \frac{\Psi_{1}(u=0) \Psi_{2}(u=0)}{\sqrt{\left(x-a / 2-u_{2}\right)^{2}+y^{2}+z^{2}}} d x d y d z .
\end{aligned}
$$

To evaluate $t_{V_{1}}$, let us define $p=x+a / 2-u_{1}$ and use the symmetry relation $u_{1}=-u / 2$, so that we can derive the kernel:

$$
\frac{\partial t_{V_{1}}}{\partial u}=\int_{-\infty}^{\infty} \frac{\partial\left[\Psi_{1}(p, u, y, z) \Psi_{2}(p, u, y, z)\right]}{\partial u} V_{1}(p, y, z) d p d y d z .
$$

Next we notice that

$$
\lim _{u \rightarrow 0} \frac{\partial\left[\Psi_{1}(p, u, y, z) \Psi_{2}(p, u, y, z)\right]}{\partial u}=-\frac{1}{2} \lim _{u \rightarrow 0} \frac{\partial\left[\Psi_{1}(p, u, y, z) \Psi_{2}(p, u, y, z)\right]}{\partial p}=-\frac{1}{2} \frac{\partial\left(\Psi_{1} \Psi_{2}\right)}{\partial x},
$$

so that finally we can write:

$$
\lim _{u \rightarrow 0} \frac{\partial t_{V_{1}}}{\partial u}=-\frac{1}{2} \int_{-\infty}^{\infty} \frac{\partial\left[\Psi_{1} \Psi_{2}\right]}{\partial x} V_{1} d x d y d z .
$$

By performing similar manipulation on $t_{V_{2}}\left(u_{2}\right)$, but now with $u_{2}=u / 2$, we arrive at:

$$
\lim _{u \rightarrow 0} \frac{\partial t_{V_{2}}}{\partial u}=\frac{1}{2} \int_{-\infty}^{\infty} \frac{\partial\left[\Psi_{1} \Psi_{2}\right]}{\partial x} V_{2} d x d y d z
$$

Summing the two contributions yields:

$$
\gamma_{12}=-\frac{1}{2} \int_{-\infty}^{\infty} d x d y d z\left[\frac{\partial\left(\Psi_{1} \Psi_{2}\right)}{\partial x}\right] V_{1}+\frac{1}{2} \int_{-\infty}^{\infty} d x d y d z\left[\frac{\partial\left(\Psi_{1} \Psi_{2}\right)}{\partial x}\right] V_{2} \equiv-\mathcal{X}+\mathcal{Y}
$$


The first integral, $\mathcal{X}$, after explicitating $\Psi_{1}$ and $\Psi_{2}$ becomes:

$$
\mathcal{X} \equiv \frac{1}{2} \int_{-\infty}^{\infty} d x d y d z \frac{\partial}{\partial x}\left[A B\left(\phi_{1}^{2}+\phi_{2}^{2}\right)+\left(A^{2}+B^{2}\right) \phi_{1} \phi_{2}\right] V_{1}(x, y, z) .
$$

The derivatives:

$\frac{\partial\left(\phi_{1} \phi_{2}\right)}{\partial x}=-4 \Gamma^{2} x \phi_{1} \phi_{2}, \quad \frac{\partial \phi_{1}^{2}}{\partial x}=-4 \Gamma^{2}\left(x+\frac{a}{2}\right) \phi_{1}^{2}, \quad \frac{\partial \phi_{2}^{2}}{\partial x}=-4 \Gamma^{2}\left(x-\frac{a}{2}\right) \phi_{2}^{2}$.

when substituted into Eq.(37) yield

$\mathcal{X}=-2 \Gamma^{2}\left\{A B\left[\left\langle\phi_{1}\left|\left(x+\frac{a}{2}\right) V_{1}\right| \phi_{1}\right\rangle+\left\langle\phi_{2}\left|\left(x-\frac{a}{2}\right) V_{1}\right| \phi_{2}\right\rangle\right]+\left(A^{2}+B^{2}\right)\left\langle\phi_{1}\left|x V_{1}\right| \phi_{2}\right\rangle\right\}$.

Similarly we get

$$
\begin{gathered}
\mathcal{Y} \equiv \frac{1}{2} \int_{-\infty}^{\infty} d x d y d z\left[\frac{\partial\left(\Psi_{1} \Psi_{2}\right)}{\partial x}\right] V_{2}, \\
\mathcal{Y}=-2 \Gamma^{2}\left\{A B\left[\left\langle\phi_{1}\left|\left(x+\frac{a}{2}\right) V_{2}\right| \phi_{1}\right\rangle+\left\langle\phi_{2}\left|\left(x-\frac{a}{2}\right) V_{2}\right| \phi_{2}\right\rangle\right]+\left(A^{2}+B^{2}\right)\left\langle\phi_{1}\left|x V_{2}\right| \phi_{2}\right\rangle\right\} .
\end{gathered}
$$

The contributions from $\left\langle\phi_{1}\left|(x+a / 2) V_{1}\right| \phi_{1}\right\rangle$ in Eq.39 and $\left\langle\phi_{2}\left|(x-a / 2) V_{2}\right| \phi_{2}\right\rangle$ in Eq.40 vanish due to the parity of the kernel. Substituting the matrix elements from Appendix 2 we get:

$$
\begin{aligned}
\gamma_{12} & =4 \sqrt{\frac{2}{\pi}}\left(\frac{\Gamma}{a}\right)\left\{\frac{A B}{2}\left[S_{0}^{4}-\left(1-4 a^{2} \Gamma^{2}\right) F_{0}\left(2 a^{2} \Gamma^{2}\right)\right]\right\} \\
& +4 \sqrt{\frac{2}{\pi}}\left(\frac{\Gamma}{a}\right)\left\{\left(A^{2}+B^{2}\right) S_{0}\left[S_{0}-F_{0}\left(a^{2} \Gamma^{2} / 2\right)\right]\right\} .
\end{aligned}
$$

Under site permutation $a \rightarrow-a$ and $A \rightarrow B$ so that $\gamma_{12}=-\gamma_{21 n a}$ as expected.

In conclusion, in the non-adiabatic limit the electron-phonon Hamiltonian is given by:

$$
\begin{aligned}
H_{e l-p h o n}^{n a}= & g_{0} \sum_{\sigma}\left(n_{1 \sigma} u_{1}+n_{2 \sigma} u_{2}\right)+g_{c f} \sum_{\sigma}\left(n_{1 \sigma} u_{2}+n_{2 \sigma} u_{1}\right) \\
& +\gamma_{12} \sum_{\sigma}\left(c_{1 \sigma}^{\dagger} c_{2 \sigma}+c_{2 \sigma}^{\dagger} c_{1 \sigma}\right)\left(u_{2}-u_{1}\right) .
\end{aligned}
$$

\section{Couplings in the adiabatic limit}

One can use the method introduced in the non-adiabatic limit to evaluate the couplings in the adiabatic case. However, one readily verifies that, in the explicit expression of the different electronic interactions in the adiabatically displaced state, $u$ invariably enters in the combination $a+u$. Therefore the procedure of first deriving the integral kernels with respect to $u$, then taking the limit $u \rightarrow 0$, and finally evaluating the integrals is equivalent to first evaluating the interactions for 
$u=0$, and then deriving them with respect to $a$. All the parameters in Eq.11 were explicitly evaluated in Ref.1, so we shall simply derive them with respect to the dimer length $a$.

\subsection{The coupling term derived from $\epsilon$}

There is some confusion in the literature about the correct form of the electronphonon Hamiltonian obtained in this limit from the variation of the local energy $\epsilon$, therefore we shall devote some space to discussing this point.

In the adiabatic limit, $u_{i} \neq 0$ in both the charge distributions and in the potentials. As the origin of the $x$-coordinate can be placed onto one of the displaced ions, this interaction couples the charge on site $i$ to the position of site $j$ through the modification of both the kinetic and the potential contributions. One has then to take into account the relative displacement of the ions. To obtain coupling terms from $\epsilon_{\nabla}$ and $\epsilon_{V_{i}}^{(i)}$ it is essential that one uses the proper Wannier functions. If one instead adopted the local orbitals, such couplings would vanish. In the adiabatic limit, therefore, the overall $\epsilon$-derived electron-phonon coupling term in the Hamiltonian is:

$$
g_{\epsilon}^{(1)} \sum_{\sigma} n_{1 \sigma}\left(u_{2}-u_{1}\right)+g_{\epsilon}^{(2)} \sum_{\sigma} n_{2 \sigma}\left(u_{1}-u_{2}\right) .
$$

As $g_{\epsilon}^{(1)}=g_{\epsilon}^{(2)}$ we can drop the site indexes, and write the total adiabatic contribution from local energy terms to the electron-phonon Hamiltonian as:

$$
H_{e l-p h o n}^{\epsilon}=g_{\epsilon} \sum_{\sigma}\left(n_{2 \sigma}-n_{1 \sigma}\right)\left(u_{1}-u_{2}\right) .
$$

An expression similar to Eq.44 has been proposed in Ref.7. To convince the skeptical reader, in Appendix 4 we show, using the contribution from $\epsilon_{V_{2}}^{(1)}$ as an example, that, starting from the general definition of the coupling, one verifies Eq.44.

\subsection{Explicit expressions for the adiabatic couplings.}

\subsubsection{Couplings originated from one-body electronic interactions.}

There are two type of couplings: one $\left(g_{\epsilon}\right)$, derived from the local energy:

$$
g_{\epsilon} \equiv g_{\nabla}^{(i)}+g_{V_{i}}^{(i)}+g_{V_{j}}^{(i)} \quad(i, j=1,2),
$$

and one $\left(\gamma_{12}\right)$ from the hopping term (Su-Schrieffer-Heeger coupling):

$$
\gamma_{12} \equiv \gamma_{12 \nabla}+\gamma_{12 V}
$$

Below we list each contribution to the electronic interactions and the derived electronphonon couplings. From the local energy on site 1

$$
\epsilon^{(1)} \equiv \epsilon_{\nabla}+\epsilon_{V_{1}}^{(1)}+\epsilon_{V_{2}}^{(1)},
$$




$$
\begin{gathered}
\epsilon_{\nabla}=\frac{\hbar^{2}}{2 m}\left[3 \Gamma^{2}+\Gamma^{4}\left(\frac{a^{2} S^{2}}{\left.1-S^{2}\right)}\right)\right], \\
\epsilon_{V_{1}}^{(1)}=-Z e^{2}\left(2 \Gamma \sqrt{\frac{2}{\pi}}\right)\left[A^{2}+B^{2} F_{0}\left(2 a^{2} \Gamma^{2}\right)+2 A B S F_{0}\left(a^{2} \Gamma^{2} / 2\right)\right], \\
\epsilon_{V_{2}}^{(1)}=-Z e^{2}\left(2 \Gamma \sqrt{\frac{2}{\pi}}\right)\left[B^{2}+A^{2} F_{0}\left(2 a^{2} \Gamma^{2}\right)+2 A B S F_{0}\left(a^{2} \Gamma^{2} / 2\right)\right],
\end{gathered}
$$

one obtains the three contributions to

$$
\begin{gathered}
g_{\epsilon}^{(1)} \equiv g_{\nabla}+g_{V_{1}}^{(1)}+g_{V_{2}}^{(1)} \\
g_{\nabla}=-\frac{\hbar^{2}}{2 m}\left[\frac{a \Gamma^{4} S^{2}}{\left(1-S^{2}\right)^{2}}\right]\left[2\left(1-a^{2} \Gamma^{2}-S^{2}\right)\right] \\
g_{V_{1}}^{(1)}=-Z e^{2}\left(2 \Gamma \sqrt{\frac{2}{\pi}}\right)\left[\frac{\partial A^{2}}{\partial u}+\frac{2 A B S^{2}+B^{2} S^{4}}{a}+F_{0}\left(2 a^{2} \Gamma^{2}\right)\left(\frac{\partial B^{2}}{\partial u}-\frac{B^{2}}{a}\right)\right], \\
\left.-Z e^{2}\left(2 \Gamma \sqrt{\frac{2}{\pi}}\right)(2 S)\left[\frac{\partial A B}{\partial u}-\frac{A B}{a}\left(1+a^{2} \Gamma^{2}\right)\right)\right] \\
g_{V_{2}}^{(1)}=-Z e^{2}\left(2 \Gamma \sqrt{\frac{2}{\pi}}\right)\left[\frac{\partial B^{2}}{\partial u}+\frac{2 A B S^{2}+A^{2} S^{4}}{a}+F_{0}\left(2 a^{2} \Gamma^{2}\right)\left(\frac{\partial A^{2}}{\partial u}-\frac{A^{2}}{a}\right)\right], \\
\left.-Z e^{2}\left(2 \Gamma \sqrt{\frac{2}{\pi}}\right)(2 S)\left[\frac{\partial A B}{\partial u}-\frac{A B}{a}\left(1+a^{2} \Gamma^{2}\right)\right)\right] .
\end{gathered}
$$

The two potential contributions depend on the site for which they are evaluated, but their sum, $g_{V_{1}}^{(1)}+g_{V_{2}}^{(1)}$, does not.

For the hopping term, we can lump together the potential contributions which individually have no particular physical meaning. Then from

$$
\begin{gathered}
t \equiv t_{\nabla}+t_{V}, \\
t_{\nabla}=\frac{\hbar^{2}}{2 m} \frac{a^{2} S \Gamma^{4}}{\left(1-S^{2}\right)}, \\
t_{V}=-Z e^{2}\left(2 \Gamma \sqrt{\frac{2}{\pi}}\right)\left\{2 A B\left[1+F_{0}\left(2 a^{2} \Gamma^{2}\right)\right]+2\left(A^{2}+B^{2}\right) S F_{0}\left(a^{2} \Gamma^{2} / 2\right)\right\},
\end{gathered}
$$

the Su-Schrieffer-Heeger coupling $\gamma_{12} \equiv \gamma_{12 \nabla}+\gamma_{12 V}$ is obtained as:

$$
\begin{gathered}
\gamma_{12 \nabla}=\frac{\hbar^{2}}{2 m}\left[\frac{a S \Gamma^{4}}{\left(1-S^{2}\right)^{2}}\right]\left[2\left(1-S^{2}\right)-a^{2} \Gamma^{2}\left(1+S^{2}\right)\right] \\
\gamma_{12 V}=-Z e^{2}\left(4 \Gamma \sqrt{\frac{2}{\pi}}\right)\left\{\left[\frac{\partial A B}{\partial u}+\frac{A^{2}+B^{2}}{a} S^{2}+\frac{A B}{a} S^{4}\right]+\left[\frac{\partial A B}{\partial u}-\frac{A B}{a}\right] F_{0}\left(2 a^{2} \Gamma^{2}\right),\right.
\end{gathered}
$$




$$
\left.+S\left[\frac{\partial\left(A^{2}+B^{2}\right)}{\partial u}-\frac{A^{2}+B^{2}}{a}\left(1+a^{2} \Gamma^{2}\right)\right] F_{0}\left(a^{2} \Gamma^{2} / 2\right)\right\} .
$$

Notice that, as the partial derivatives are linear in $a$, then both $\gamma_{12 \nabla}$ and $\gamma_{12 V}$ change sign under site permutation, as expected from Refs.2,3.

The electron-phonon Hamiltonian in the adiabatic limit has therefore the form:

$$
H_{\text {el-phon }}^{a d}=g_{\epsilon} \sum_{\sigma}\left(n_{1 \sigma}-n_{2 \sigma}\right)\left(u_{2}-u_{1}\right)+\gamma_{12} \sum_{\sigma}\left(c_{1 \sigma}^{\dagger} c_{2 \sigma}+c_{2 \sigma}^{\dagger} c_{1 \sigma}\right)\left(u_{2}-u_{1}\right) .
$$

\subsubsection{Couplings originated from two-body electronic interactions.}

Also the two-body electronic interactions $U, V, J(=P), X$ of Eq. (1) give origin to electron-phonon couplings which, to the best of our knowledge, have never been considered in the literature up to now. The electron-phonon coupling originating from the modulation of the kinetic exchange in the $t-J$ Hamiltonian has been considered in Ref.17. However, the kinetic exchange $J_{k i n} \equiv 2 t^{2} /(U-V)$ is a composite quantity different from the direct exchange $J_{x y}=J_{z}=P$ in Eq. (1). Here we list the values the two-body electron-phonon interactions have in our model. They were obtained by deriving with respect to $a$ the two-body interactions evaluated explicitly in Ref.1. Notice that, as $U(a)=J(a)+e^{2} \Gamma / \sqrt{\pi}$, then $d U / d a=d J / d a=d P / d a$

$$
\begin{aligned}
\frac{d X}{d a}=- & e^{2} \frac{\Gamma}{\sqrt{\pi}}\left[(-a \Gamma S) \frac{\left(1+3 S^{2}\right)}{\left(1-S^{2}\right)^{3}}\right]\left[1+2 S^{2}+F_{0}\left(a^{2} \Gamma^{2}\right)-2\left(1+S^{2}\right) F_{0}\left(\frac{a^{2} \Gamma^{2}}{4}\right)\right] \\
-e^{2} \frac{\Gamma}{\sqrt{\pi}} & {\left[\frac{S / a}{\left(1-S^{2}\right)^{2}}\right]\left\{4 a^{2} \Gamma^{2} S^{2}\left[F_{0}\left(\frac{a^{2} \Gamma^{2}}{4}\right)-1\right]+S^{2}-F_{0}\left(a^{2} \Gamma^{2}\right)\right\} } \\
& -e^{2} \frac{\Gamma}{\sqrt{\pi}}\left[\frac{S / a}{\left(1-S^{2}\right)^{2}}\right]\left\{2\left(1+S^{2}\right)\left[F_{0}\left(\frac{a^{2} \Gamma^{2}}{4}\right)-\sqrt{S}\right]\right\} \\
\frac{d U}{d a}= & e^{2} \frac{\Gamma}{\sqrt{\pi}}\left[\frac{-4 a \Gamma^{2} S^{2}}{\left(1-S^{2}\right)^{3}}\right]\left[2-S^{2}+2 S^{4}+S^{2} F_{0}\left(a^{2} \Gamma^{2}\right)-4 S^{2} F_{0}\left(\frac{a^{2} \Gamma^{2}}{4}\right)\right] \\
+ & e^{2} \frac{\Gamma}{\sqrt{\pi}}\left[\frac{S^{2} / a}{\left(1-S^{2}\right)^{2}}\right]\left\{2 a^{2} \Gamma^{2}\left[1-4 S^{2}-F_{0}\left(a^{2} \Gamma^{2}\right)+4 F_{0}\left(\frac{a^{2} \Gamma^{2}}{4}\right)\right]\right. \\
\frac{d V}{d a}= & e^{2} \frac{\Gamma}{\sqrt{\pi}}\left[-\frac{4 a \Gamma^{2} S^{2}}{\left(1-S^{2}\right)^{3}}\right]\left[3-S^{2}-8 S^{4}-\left(7-5 S^{2}\right) F_{0}\left(a^{2} \Gamma^{2}\right)-4\left(1-3 S^{2}\right) F_{0}\left(\frac{a^{2} \Gamma^{2}}{4}\right)\right] \\
+ & e^{2} \frac{\Gamma}{\sqrt{\pi}}\left[\frac{1 / a}{\left(1-S^{2}\right)^{2}}\right]\left\{2 a^{2} \Gamma^{2} S^{2}\left[-1-4 S^{2}+F_{0}\left(a^{2} \Gamma^{2}\right)+4 F_{0}\left(\frac{a^{2} \Gamma^{2}}{4}\right)\right]\right.
\end{aligned}
$$




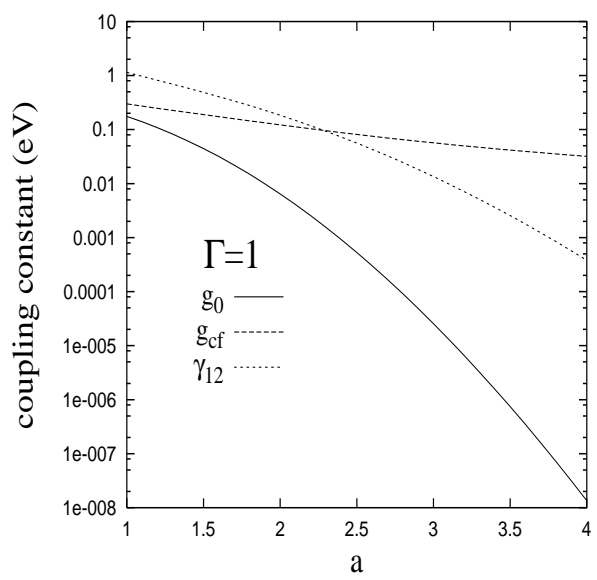

Figure 1: Non-adiabatic coupling constants $g_{0}, g_{c f}, \gamma_{12}$ (in $\mathrm{eV}$ ) versus the dimer length (in $\AA$ ), evaluated assuming $\Gamma=1.0 \AA^{-1}$.

$$
\begin{gathered}
\left.+2 S^{2}+\frac{3}{2} S^{4}-\left(2-\frac{3}{2} S^{2}\right) F_{0}\left(a^{2} \Gamma^{2}\right)+2 S^{2}\left[F_{0}\left(\frac{a^{2} \Gamma^{2}}{4}\right)-\sqrt{S}\right]\right\}, \\
\frac{d J}{d a} \equiv \frac{d P}{d a} \equiv \frac{d U}{d a} .
\end{gathered}
$$

All the above interactions change sign under site permutation. The terms they contribute to the electron-phonon Hamiltonian all have the same form, namely:

$$
H_{Y}^{e p}=\frac{d Y}{d a} F\left(c_{i \sigma}^{\dagger}, c_{j \sigma}\right)\left(u_{j}-u_{j}\right) \quad(i, j=1,2)
$$

where $Y=U, V, X, J$ and $F\left(c_{i \sigma}^{\dagger}, c_{j \sigma}\right)(i, j=1,2)$ is the function of Fermi operators representing the two-body interaction whose amplitude is $Y$

\section{Results}

By definition, as $n_{i \sigma}$ is the number of electrons per site (a dimensionless quantity), then the interaction parameters have dimensions [energy][length $]^{-1}$. To get them in energy units $(\mathrm{eV})$ we shall measure the deformations $u_{i}$ in units of the characteristic phonon length $L=\sqrt{\hbar /(2 \Omega M)}$. We choose the phonon frequency such that $\hbar \Omega=0.1 \mathrm{eV}$, which is appropriate to HTS and $C M R$, and $M$ equal to the mass of ${ }^{16} \mathrm{O}$. Figs. 1 and 2 show the behaviour versus the dimer length $a$ (in $\AA$ ) of the non-adiabatic coupling parameters $g_{0}, g_{c f}, \gamma_{12}$ (in $\mathrm{eV}$ ) evaluated by assuming two representative values $\Gamma=1.0,2.0 \AA^{-1}$ for the shape-controlling parameter of the Wannier functions. The most unexpected result concerns $g_{c f}$. While usually neglected in the literature on metallic systems, this coupling has been recognized as relevant to polar materials. than $g_{0}$ for any $a$, and it becomes the largest parameter for $a>2.2 \AA$. For small $a$, 


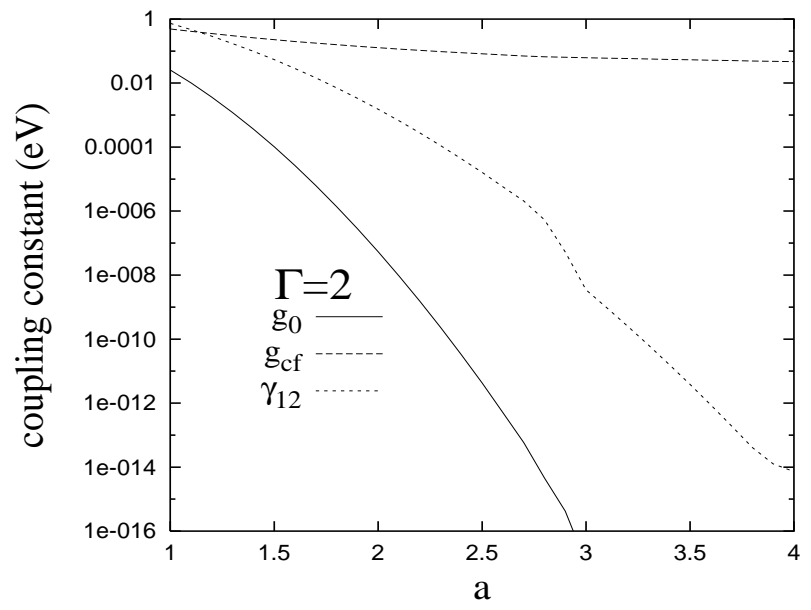

Figure 2: Non-adiabatic coupling constants $g_{c f a d}, \gamma_{12 a d}$ (in $\mathrm{eV}$ ) versus the dimer length (in $\AA$ ), for $\Gamma=2.0 \AA^{-1}$.

the $S S H$ coupling is the largest. The strength of all the couplings decreases with $a$, less quickly for $g_{c f}$, which, for large $a$, is still comparable to the values of the hopping amplitude $t(a)$.

When $\Gamma=2.0 \AA^{-1}$ (see Fig.2) $g_{0}$ is negligible for any $a, \gamma_{12}$ is large for small $a$ but drops very rapidly to negligible values as $a$ increases, while $g_{c f}$ keeps appreciable values for all $a$ values. We can conclude that, in the non-adiabatic limit, the more localized are the orbitals, the more relevant is the role of $g_{c f}$ in relation to the other admissible couplings.

Fig.3 is the adiabatic counterpart of Fig.1. The Holstein type interaction is absent, being identically vanishing as discussed above. Here we find that $g_{\epsilon}$ is always larger than the $S S H$ interaction $\gamma_{12}$, and particularly for large $a$ there is an order of magnitude difference between them.

Fig.4 shows the couplings derived from the two-body electronic interactions for the same parameters as Fig.3. In general, their values are smaller than those of $g_{\epsilon}$ and $\gamma_{12}$, with the possible exception of $d V / d a$. Indeed, that coupling arises from a physical mechanism not very different from the one originating $g_{V_{j}}^{(i)}$, i.e. the vibration of the charge on site $j$ as felt by site $i$. Similarly to $g_{\epsilon}$ also $d V / d a$ decreases slowly with $a$, so that for large $a$ those two are the only relevant couplings. It is worth stressing that, though $U$ is larger than $J$, their derivatives coincide. Besides, the derivative of the interaction coupling $X$ is a non-monotonic function of the lattice constant. Indeed, $X$ exhibits a maximum for $a \simeq 1.8$ and then sharply decreases to a negligible value.

When $\Gamma=2.0 \AA^{-1}$, as shown in Fig.5, $g_{\epsilon}$ and $\gamma_{12}$ have trends similar to the nonadiabatic case, with larger values for small $a$. As $a$ increases, $\gamma_{12}$ quickly reduces to negligible values, while $g_{\epsilon}$ decreases more slowly. Of the two-body couplings, only $d V / d a$ is non-negligible, and it has a strength very close (in absolute value) to $g_{\epsilon}$. 


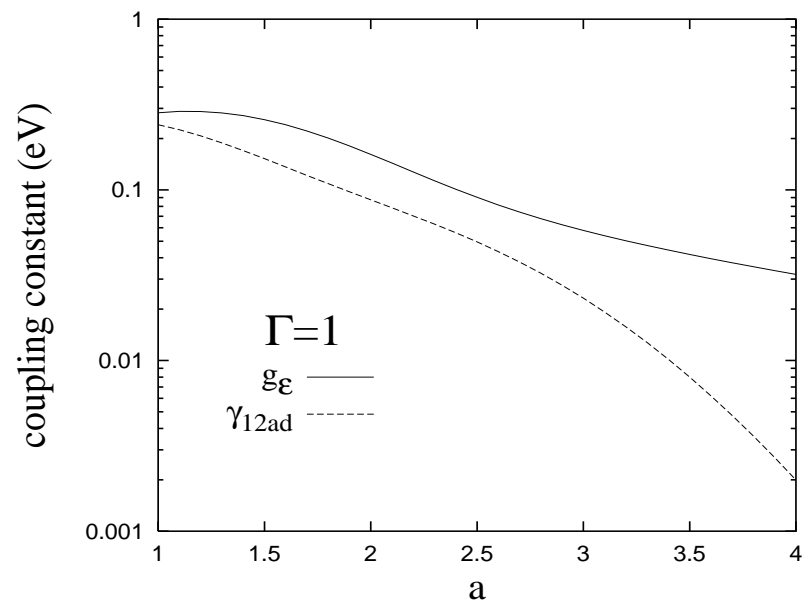

Figure 3: Adiabatic coupling constants $g_{\epsilon}, \gamma_{12 a d}$ (in $\mathrm{eV}$ ) versus the dimer length (in $\AA)$, for $\Gamma=1.0 \AA^{-1}$.

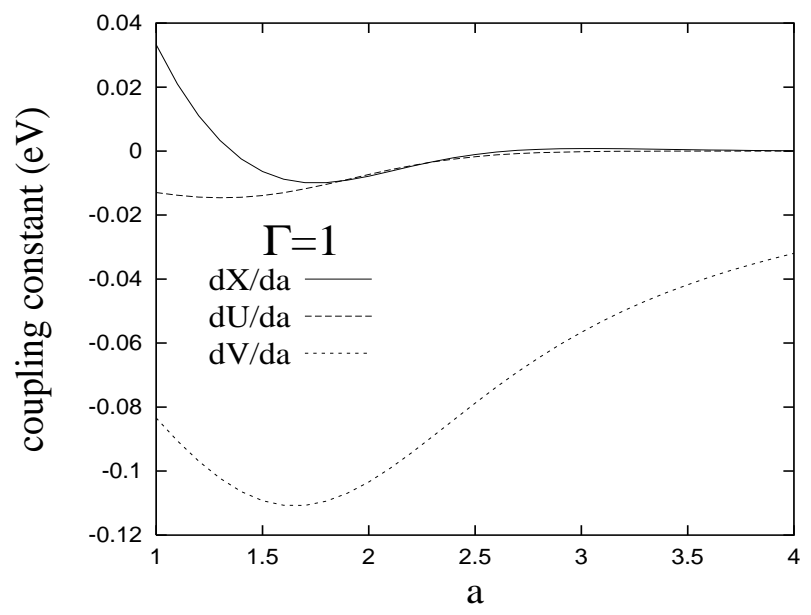

Figure 4: Adiabatic coupling constants from two-body interactions $d X / d a, d U / d a, d V / d a$ (in $\mathrm{eV}$ ) versus the dimer length (in $\AA)$, for $\Gamma=1.0 \AA^{-1}$. 


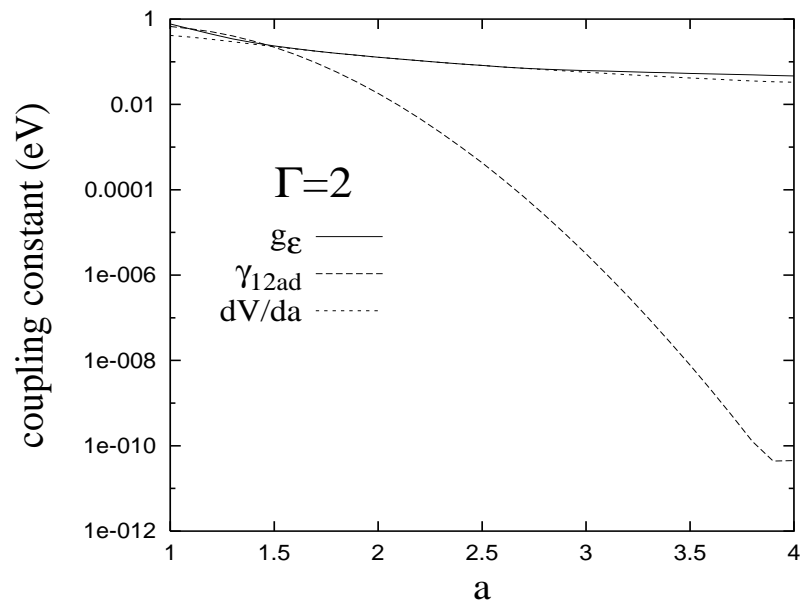

Figure 5: Adiabatic coupling constants $g_{\epsilon}, \gamma_{12}, d V / d a$ (in $\mathrm{eV}$ ) versus the dimer length (in $\AA$ ), for $\Gamma=2.0 \AA^{-1}$.

\section{Conclusions}

We have presented the analytical evaluation of the electron-phonon coupling parameters derived from both one- and two-body electronic interactions in a model of a dimer with a non-degenerate orbital built from atomic orbital of Gaussian shape. The approach we have followed (proposed in general terms in Refs.2,3) consists in inserting the site displacements $u_{1}, u_{2}$ in the kernels of the integrals defining the one-body electronic parameters $\epsilon_{0}$ and $t$, and then considering the first-order terms in the expansion of $\epsilon_{0}\left(u_{1}, u_{2}\right)$ and $t\left(u_{1}, u_{2}\right)$. In the adiabatic case this procedure is equivalent to the simpler on 2 3. 12 consisting in evaluating the electronic parameters as functions of the lattice parameter (corresponding to the dimer length $a$ ) and then deriving with respect to $a$. In the anti-adiabatic case, however, the latter procedure can not be applied, because $a$ and $u$ enter independently the kernel of the integrals. From the physical point of view, the " $a$-derivative" method describes how the electronic parameters vary under a quasi-static (low frequency) change of the equilibrium position of the ions, as could be realized e.g. under pressure. In general the electron-phonon couplings are the effect of the ion oscillations at frequency $\Omega$, which causes the system to be alternatively compressed and elongated over a time $\approx \Omega^{-1}$. It is this implicit dynamics of the displacements which allows for distinguishing between the adiabatic and non-adiabatic regimes, and forbids the use of the "a-derivative" method in the latter case.

A novel result is the evaluation of the couplings originating from the two-body electronic interactions. We have shown that at least one of them, generated by the Coulomb repulsion between the charges on different sites, is comparable, or even larger, than the couplings derived from the one-body interactions.

The quantitative results for the coupling parameters, even if agreeing in order 
of magnitude with some estimates from experimental data (see, e.g. Ref.13) are obviously model-depending. However, their ratios should be more close to the reality. In particular, the obtained values of the various couplings, when compared to the values of the electronic interactions obtained from the same Wannier functiond suggests that, for dimer lengths comparable to the lattice parameters in $H T S$ and $C M R$, only $d U / d a$ and $d X / d a$ can be safely dropped, while neglecting any of the other electron-phonon interactions is a questionable approximation.

Finally, we have been able to determine the correct form of the admissible electron-phonon coupling one-body terms in the interacting Hamiltonian. In the literature one sometimes finds proposals for such terms which are incompatible with our results. This is the case, for instance, of the electron-phonon Hamiltonian of Refs.14,15.

\section{Acknowledgements}

It is a pleasure to thank J.R. Iglesias, M. A. Gusmão, A. Painelli, M. Cococcioni, A. Alexandrov, and particularly A.A. Aligia, for critical discussions and comments. This work was supported by I.N.F.M. and by MURST 1997 co-funded project "Magnetic Polarons in Manganites" . 


\section{Appendix 1}

Here we list some results of frequent use in the calculations. We shall use the zero-displacement derivatives of $A$ and $B$ :

$$
\begin{aligned}
& \left.\frac{\partial A}{\partial u}\right|_{u \rightarrow 0}=\frac{a \Gamma^{2} S_{0}}{4}\left[-\left(1+S_{0}\right)^{-3 / 2}+\left(1-S_{0}\right)^{-3 / 2}\right], \\
& \left.\frac{\partial B}{\partial u}\right|_{u \rightarrow 0}=\frac{a \Gamma^{2} S_{0}}{4}\left[-\left(1+S_{0}\right)^{-3 / 2}-\left(1-S_{0}\right)^{-3 / 2}\right] .
\end{aligned}
$$

In the following we shall use the substitution $t=x+a / 2-u_{1}$ in both $\Psi_{1}$ and $\Psi_{2}$, yielding:

$$
\begin{aligned}
& \Psi_{1}\left(x, u_{1}, u_{2}, y, z\right) \Rightarrow \Psi_{1}(t, u, y, z)=A(u) N e^{-\Gamma^{2}\left[t^{2}+y^{2}+z^{2}\right]}+B(u) N e^{-\Gamma^{2}\left[(t-a+u)^{2}+y^{2}+z^{2}\right]}, \\
& \Psi_{2}\left(x, u_{1}, u_{2}, y, z\right) \Rightarrow \Psi_{2}(t, u, y, z)=B(u) N e^{-\Gamma^{2}\left[t^{2}+y^{2}+z^{2}\right]}+A(u) N e^{-\Gamma^{2}\left[(t-a+u)^{2}+y^{2}+z^{2}\right]} .
\end{aligned}
$$

To write down their derivatives in the zero-displacement limit, it is convenient to revert to $x=t-a / 2$ so that:

$$
\begin{aligned}
& \left.\frac{\partial \Psi_{1}(x, y, z, u)}{\partial u}\right|_{u \rightarrow 0}=\phi_{1}(x, y, z) \frac{\partial A}{\partial u}+\phi_{2}(x, y, z)\left[\frac{\partial B}{\partial u}-2 \Gamma^{2}\left(x-\frac{a}{2}\right) B\right], \\
& \left.\frac{\partial \Psi_{2}(x, y, z, u)}{\partial u}\right|_{u \rightarrow 0}=\phi_{1}(x, y, z) \frac{\partial B}{\partial u}+\phi_{2}(x, y, z)\left[\frac{\partial A}{\partial u}-2 \Gamma^{2}\left(x-\frac{a}{2}\right) A\right] .
\end{aligned}
$$

We shall also use the other substitution $t \equiv x-a / 2-u_{2}$, yielding:

$$
\begin{gathered}
\Psi_{1}\left(x, u_{1}, u_{2},, y, z\right) \Rightarrow A(u) N e^{-\Gamma^{2}\left[(t+a-u)^{2}+y^{2}+z^{2}\right]}+B(u) N e^{-\Gamma^{2}\left[t^{2}+y^{2}+z^{2}\right]}, \\
\Psi_{2}\left(x, u_{1}, u_{2}, y, z\right) \Rightarrow B(u) N e^{-\Gamma^{2}\left[(t+a-u)^{2}+y^{2}+z^{2}\right]}+A(u) N e^{-\Gamma^{2}\left[t^{2}+y^{2}+z^{2}\right]} .
\end{gathered}
$$

In the limit of vanishing deformations it follows, after reverting to $x=t+a / 2$ :

$$
\begin{aligned}
& \left.\frac{\partial \Psi_{1}(x, y, z, u)}{\partial u}\right|_{u \rightarrow 0}=\phi_{1}(x, y, z)\left[\frac{\partial A}{\partial u}+2 \Gamma^{2}\left(x+\frac{a}{2}\right) A\right]+\phi_{2}(x, y, z) \frac{\partial B}{\partial u}, \\
& \left.\frac{\partial \Psi_{2}(x, y, z, u)}{\partial u}\right|_{u \rightarrow 0}=\phi_{1}(x, y, z)\left[\frac{\partial B}{\partial u}+2 \Gamma^{2}\left(x+\frac{a}{2}\right) B\right]+\phi_{2}(x, y, z) \frac{\partial A}{\partial u} .
\end{aligned}
$$

\section{Appendix 2}

For convenience reasons we shall list here various matrix elements, all evaluated in the limit of vanishing deformations, which enter the calculations. Those of 
them which do not follow straightforwardly from standard properties of Gaussian integrals 16 are evaluated in details in the Appendix 3. By defining:

$$
F_{0}(x)=\frac{1}{\sqrt{x}} \int_{0}^{\sqrt{x}} e^{-t^{2}} d t
$$

we have:

$$
\begin{gathered}
\left\langle\phi_{i}\left|-\nabla^{2} / 2\right| \phi_{i}\right\rangle=\frac{3 \Gamma^{2}}{2}+\frac{\Gamma^{4} a^{2} S_{0}^{2}}{2\left(1-S_{0}^{2}\right)}, \quad\left\langle\phi_{i}\left|-\nabla^{2} / 2\right| \phi_{j}\right\rangle=-\frac{\Gamma^{4} a^{2} S_{0}}{2\left(1-S_{0}^{2}\right)}, \\
\left\langle\phi_{i}\left|V_{i}\right| \phi_{i}\right\rangle=2 \Gamma \sqrt{2 / \pi}, \quad\left\langle\phi_{i}\left|V_{j}\right| \phi_{i}\right\rangle=2 \Gamma \sqrt{2 / \pi} F_{0}\left(2 a^{2} \Gamma^{2}\right) \\
\left\langle\phi_{i}\left|V_{i}\right| \phi_{j}\right\rangle=2 \Gamma \sqrt{2 / \pi} S_{0} F_{0}\left(\frac{a^{2} \Gamma^{2}}{2}\right) \\
\left\langle\phi_{1}\left|x V_{1}\right| \phi_{1}\right\rangle=-a \Gamma \sqrt{2 / \pi}, \quad\left\langle\phi_{2}\left|x V_{2}\right| \phi_{2}\right\rangle=a \Gamma \sqrt{2 / \pi} \\
\left\langle\phi_{1}\left|x V_{2}\right| \phi_{1}\right\rangle=\frac{1}{2 a \Gamma} \sqrt{\frac{2}{\pi}}\left[-S_{0}^{4}+\left(1-2 a^{2} \Gamma^{2}\right) F_{0}\left(2 a^{2} \Gamma^{2}\right)\right] \\
\left\langle\phi_{2}\left|x V_{1}\right| \phi_{2}\right\rangle=-\left\langle\phi_{1}\left|x V_{2}\right| \phi_{1}\right\rangle \\
\left\langle\phi_{1}\left|x V_{1}\right| \phi_{2}\right\rangle=\frac{S_{0} \sqrt{2 / \pi}}{a \Gamma}\left[S_{0}-F_{0}\left(\frac{a^{2} \Gamma^{2}}{2}\right)\right] \\
\left\langle\phi_{1}\left|x V_{2}\right| \phi_{2}\right\rangle=-\left\langle\phi_{1}\left|x V_{1}\right| \phi_{2}\right\rangle
\end{gathered}
$$

Some other useful relations are:

$$
\left\langle\phi_{1}\left|V_{1}\right| \phi_{1}\right\rangle=\left\langle\phi_{2}\left|V_{2}\right| \phi_{2}\right\rangle, \quad\left\langle\phi_{2}\left|V_{1}\right| \phi_{2}\right\rangle=\left\langle\phi_{1}\left|V_{2}\right| \phi_{1}\right\rangle .
$$

We can directly evaluate

$$
\left\langle\phi_{1}\left|x V_{1}\right| \phi_{1}\right\rangle=-a \Gamma \sqrt{2 / \pi}=-\left\langle\phi_{2}\left|x V_{2}\right| \phi_{2}\right\rangle .
$$

Indeed, due to the odd parity of the kernel, $\left\langle\phi_{1}\left|(x+a / 2) V_{1}\right| \phi_{1}\right\rangle=0$ from which Eq.(72) follows. We can also prove that

$$
\left\langle\phi_{1}\left|x V_{2}\right| \phi_{2}\right\rangle=-\left\langle\phi_{2}\left|x V_{1}\right| \phi_{1}\right\rangle, \quad\left\langle\phi_{1}\left|x V_{2}\right| \phi_{1}\right\rangle=-\left\langle\phi_{2}\left|x V_{1}\right| \phi_{2}\right\rangle .
$$

Indeed, if $x$ changes sign, then $V_{1} \rightarrow V_{2}$ and $\phi_{1} \rightarrow \phi_{2}$. Therefore the functions $\phi_{1}\left(V_{1}+V_{2}\right) \phi_{2}$ and $\phi_{1}^{2} V_{2}+\phi_{2}^{2} V_{1}$ are even in $x$, so that:

$$
\begin{gathered}
\left\langle\phi_{1}\left|x V_{2}\right| \phi_{2}\right\rangle+\left\langle\phi_{2}\left|x V_{1}\right| \phi_{1}\right\rangle=\left\langle\phi_{1}\left|x\left(V_{1}+V_{2}\right)\right| \phi_{2}\right\rangle=0, \\
\left\langle\phi_{1}\left|x V_{2}\right| \phi_{1}\right\rangle+\left\langle\phi_{2}\left|x V_{1}\right| \phi_{2}\right\rangle=\int_{-\infty}^{\infty} x\left(\phi_{1}^{2} V_{2}+\phi_{2}^{2} V_{1}\right) d x d y d z=0,
\end{gathered}
$$

because the integrands are odd functions of $\mathrm{x}$. 


\section{Appendix 3}

Let us now evaluate the unknown integrals in Eq.(20). To do that, consider

$$
\left\langle\Psi_{1}\left|V_{1}\right| \Psi_{1}\right\rangle=\int_{-\infty}^{\infty} \Psi_{1}(a / 2, x, y, z)^{2} V_{1}(a / 2, x, y, z) d x d y d z,
$$

and its derivative with respect to $a / 2$ :

$$
\begin{aligned}
\frac{\partial\left\langle\Psi_{1}\left|V_{1}\right| \Psi_{1}\right\rangle}{\partial(a / 2)}= & 2 \int_{-\infty}^{\infty} \Psi_{1}\left[\frac{\partial \Psi_{1}}{\partial(a / 2)}\right] V_{1} d x d y d z+\int_{-\infty}^{\infty} \Psi_{1}^{2} \frac{\partial V_{1}}{\partial(a / 2)} d x d y d z \\
& =2 \int_{-\infty}^{\infty} \Psi_{1}\left[\frac{\partial \Psi_{1}}{\partial(a / 2)}\right] V_{1} d x d y d z-g_{0}^{(1)},
\end{aligned}
$$

where we use the Eq.(19) and $\lim _{u_{1} \rightarrow 0} \partial V_{1} / \partial u_{1}=-\partial V_{1} / \partial x=-\partial V_{1} / \partial(a / 2)$. To evaluate the first integral we need also:

$$
\frac{\partial \Psi_{1}}{\partial(a / 2)}=\frac{\partial A}{\partial(a / 2)} \phi_{1}+\frac{\partial B}{\partial(a / 2)} \phi_{2}-2 \Gamma^{2}\left[A(x+a / 2) \phi_{1}-B(x-a / 2) \phi_{2}\right] .
$$

Inside the integral, it is convenient to use $\partial / \partial x$, instead of $\partial / \partial(a / 2)$. As

$$
\frac{\partial \Psi_{1}}{\partial x}=-2 \Gamma^{2}\left[A(x+a / 2) \phi_{1}+B(x-a / 2) \phi_{2}\right]
$$

then

$$
\frac{\partial \Psi_{1}}{\partial(a / 2)}=\frac{\partial A}{\partial(a / 2)} \phi_{1}+\frac{\partial B}{\partial(a / 2)} \phi_{2}+\frac{\partial \Psi_{1}}{\partial x}+4 \Gamma^{2} B(x-a / 2) \phi_{2},
$$

so that Eq. (75) becomes, recalling Eq.(19):

$$
\begin{gathered}
\frac{d\left\langle\Psi_{1}\left|V_{1}\right| \Psi_{1}\right\rangle}{d(a / 2)}=2 \frac{\partial A}{\partial(a / 2)} \int_{-\infty}^{\infty} \Psi_{1} \phi_{1} V_{1} d x d y d z+2 \frac{\partial B}{\partial(a / 2)} \int_{-\infty}^{\infty} \Psi_{1} \phi_{2} V_{1} d x d y d z \\
+8 \Gamma^{2} B \int_{-\infty}^{\infty} \Psi_{1}(x-a / 2) \phi_{2} V_{1} d x d y d z
\end{gathered}
$$

Developing $\Psi_{1}=A \phi_{1}+B \phi_{2}$ and reordering, one arrives at:

$$
\begin{aligned}
& 8 \Gamma^{2}\left(A B\left\langle\phi_{1}\left|x V_{1}\right| \phi_{2}\right\rangle+B^{2}\left\langle\phi_{2}\left|x V_{1}\right| \phi_{2}\right\rangle\right)=\frac{\partial\left\langle\Psi_{1}\left|V_{1}\right| \Psi_{1}\right\rangle}{\partial(a / 2)}-\frac{\partial A^{2}}{\partial(a / 2)}\left\langle\phi_{1}\left|V_{1}\right| \phi_{1}\right\rangle \\
& +\left[4 a \Gamma^{2} B^{2}-\frac{\partial B^{2}}{\partial(a / 2)}\right]\left\langle\phi_{2}\left|V_{1}\right| \phi_{2}\right\rangle+\left[4 a \Gamma^{2} A B-2 \frac{\partial(A B)}{\partial(a / 2)}\right]\left\langle\phi_{1}\left|V_{1}\right| \phi_{2}\right\rangle .
\end{aligned}
$$

This equation connects the unknown integrals on the left hand side to known ones. We need another relation, which is provided by similar manipulations on $\left\langle\Psi_{2}\left|V_{1}\right| \Psi_{2}\right\rangle$.

$$
\begin{gathered}
\frac{\partial\left\langle\Psi_{2}\left|V_{1}\right| \Psi_{2}\right\rangle}{\partial(a / 2)}=\frac{\partial}{\partial(a / 2)}\left[\int_{-\infty}^{\infty} \Psi_{2}(a / 2, x, y, z)^{2} V_{1}(a / 2, x, y, z) d x d y d z\right] \\
=2 \int_{-\infty}^{\infty} \Psi_{2} \frac{\partial \Psi_{2}}{\partial(a / 2)} V_{1} d x d y d z+\int_{-\infty}^{\infty} \Psi_{2}^{2} \frac{\partial V_{1}}{\partial(a / 2)} d x d y d z
\end{gathered}
$$


After substituting $\partial V_{1} / \partial(a / 2)=\partial V_{1} / \partial x$ the second integral is done by parts and one arrives at:

$$
\frac{\partial\left\langle\Psi_{2}\left|V_{1}\right| \Psi_{2}\right\rangle}{\partial(a / 2)}=2 \int_{-\infty}^{\infty} \Psi_{2}\left[\frac{\partial \Psi_{2}}{\partial(a / 2)}-\frac{\partial \Psi_{2}}{\partial x}\right] V_{1} d x d y d z .
$$

¿From the definition of $\Psi_{2}$ one has:

$$
\begin{gathered}
\frac{\partial \Psi_{2}}{\partial(a / 2)}=\frac{\partial B}{\partial(a / 2)} \phi_{1}+\frac{\partial A}{\partial(a / 2)} \phi_{2}-2 \Gamma^{2}\left[B(x+a / 2) \phi_{1}-A(x-a / 2) \phi_{2}\right] \\
\frac{\partial \Psi_{2}}{\partial x}=-2 \Gamma^{2}\left[B(x+a / 2) \phi_{1}+A(x-a / 2) \phi_{2}\right]
\end{gathered}
$$

so that

$$
\frac{\partial \Psi_{2}}{\partial(a / 2)}-\frac{\partial \Psi_{2}}{\partial x}=\frac{\partial B}{\partial(a / 2)} \phi_{1}+\frac{\partial A}{\partial(a / 2)} \phi_{2}+4 \Gamma^{2} A(x-a / 2) \phi_{2} .
$$

Substituting Eq.(84) into Eq.(82), developing $\Psi_{2}=B \phi_{1}+A \phi_{2}$ and reordering yields finally:

$$
\begin{aligned}
& 8 \Gamma^{2}\left(A B\left\langle\phi_{1}\left|x V_{1}\right| \phi_{2}\right\rangle+A^{2}\left\langle\phi_{2}\left|x V_{1}\right| \phi_{2}\right\rangle\right)=\frac{\partial\left\langle\Psi_{2}\left|V_{1}\right| \Psi_{2}\right\rangle}{\partial(a / 2)}-\frac{\partial B^{2}}{\partial(a / 2)}\left\langle\phi_{1}\left|V_{1}\right| \phi_{1}\right\rangle \\
& +\left[4 a \Gamma^{2} A^{2}-\frac{\partial A^{2}}{\partial(a / 2)}\right]\left\langle\phi_{2}\left|V_{1}\right| \phi_{2}\right\rangle+\left[4 a \Gamma^{2} A B-2 \frac{\partial(A B)}{\partial(a / 2)}\right]\left\langle\phi_{1}\left|V_{1}\right| \phi_{2}\right\rangle,
\end{aligned}
$$

which is the second equation needed to evaluate the integrals entering the Eq.(20) for $g_{0}^{(1)}$. For the explicit evaluation we need also:

$$
\begin{aligned}
& \left\langle\Psi_{1}\left|V_{1}\right| \Psi_{1}\right\rangle=2 \Gamma \sqrt{2 / \pi}\left[A^{2}+B^{2} F_{0}\left(2 \Gamma^{2} a^{2}\right)+2 A B S F_{0}\left(\Gamma^{2} a^{2} / 2\right)\right], \\
& \left\langle\Psi_{2}\left|V_{1}\right| \Psi_{2}\right\rangle=2 \Gamma \sqrt{2 / \pi}\left[B^{2}+A^{2} F_{0}\left(2 \Gamma^{2} a^{2}\right)+2 A B S F_{0}\left(\Gamma^{2} a^{2} / 2\right)\right] .
\end{aligned}
$$

It is convenient to subtract the Eq.(80) from Eq.(85) yielding:

$$
\begin{gathered}
8 \Gamma^{2}\left(A^{2}-B^{2}\right)<\phi_{2}\left|x V_{1}\right| \phi_{2}>=\frac{d}{d(a / 2)}\left[<\Psi_{2}\left|V_{1}\right| \Psi_{2}>-<\Psi_{1}\left|V_{1}\right| \Psi_{1}>\right] \\
+\frac{d\left(A^{2}-B^{2}\right)}{d(a / 2)}<\phi_{1}\left|V_{1}\right| \phi_{1}>+\left[4 \Gamma^{2} a\left(A^{2}-B^{2}\right)-\frac{d\left(A^{2}-B^{2}\right)}{d(a / 2)}\right]<\phi_{2}\left|V_{1}\right| \phi_{2}>,
\end{gathered}
$$

while their sum yields:

$$
\begin{gathered}
16 \Gamma^{2} A B<\phi_{1}\left|x V_{1}\right| \phi_{2}>+8 \Gamma^{2}\left(A^{2}+B^{2}\right)<\phi_{2}\left|x V_{1}\right| \phi_{2}>= \\
\frac{d}{d(a / 2)}\left[<\Psi_{2}\left|V_{1}\right| \Psi_{2}>+<\Psi_{1}\left|V_{1}\right| \Psi_{1}>\right]-\frac{d\left(A^{2}+B^{2}\right)}{d(a / 2)}<\phi_{1}\left|V_{1}\right| \phi_{1}>+
\end{gathered}
$$




$$
\begin{gathered}
+\left[4 \Gamma^{2} a\left(A^{2}+B^{2}\right)-\frac{d\left(A^{2}+B^{2}\right)}{d(a / 2)}\right]<\phi_{2}\left|V_{1}\right| \phi_{2}> \\
+\left[8 \Gamma^{2} a A B-4 \frac{d(A B)}{d(a / 2)}\right]<\phi_{1}\left|V_{1}\right| \phi_{2}>.
\end{gathered}
$$

To proceed, one has

$$
\begin{gathered}
<\Psi_{2}\left|V_{1}\right| \Psi_{2}>-<\Psi_{1}\left|V_{1}\right| \Psi_{1}>=-\Gamma \sqrt{\frac{2}{\pi}}\left(A^{2}-B^{2}\right)\left[1-F_{0}\left(2 \Gamma^{2} a^{2}\right)\right], \\
<\Psi_{2}\left|V_{1}\right| \Psi_{2}>+<\Psi_{1}\left|V_{1}\right| \Psi_{1}>= \\
\Gamma \sqrt{\frac{2}{\pi}}\left\{\left(A^{2}+B^{2}\right)\left[1+F_{0}\left(2 \Gamma^{2} a^{2}\right)\right]+4 A B S F_{0}\left(\Gamma^{2} a^{2} / 2\right)\right\} .
\end{gathered}
$$

The derivatives of interest are:

$$
\begin{gathered}
\frac{\partial S}{\partial(a / 2)}=-2 \Gamma^{2} a S \\
\frac{\partial\left(A^{2}+B^{2}\right)}{\partial(a / 2)}=-\frac{4 \Gamma^{2} a S^{2}}{\left(1-S^{2}\right)^{2}}, \quad \frac{\partial\left(A^{2}-B^{2}\right)}{\partial(a / 2)}=-\frac{2 \Gamma^{2} a S^{2}}{\left(1-S^{2}\right)^{3 / 2}}, \quad \frac{\partial A B}{\partial(a / 2)}=\frac{\Gamma^{2} a S\left(1+S^{2}\right)}{\left(1-S^{2}\right)^{2}} \\
\frac{\partial}{\partial(a / 2)}\left[F_{0}\left(2 \Gamma^{2} a^{2}\right)\right]=\frac{2}{a}\left[S^{4}-F_{0}\left(2 \Gamma^{2} a^{2}\right)\right], \quad \frac{\partial}{\partial(a / 2)}\left[F_{0}\left(\frac{\Gamma^{2} a^{2}}{2}\right)\right]=\frac{2}{a}\left[S-F_{0}\left(\frac{\Gamma^{2} a^{2}}{2}\right)\right] .
\end{gathered}
$$

Therefore we obtain:

$$
\begin{gathered}
\frac{\partial}{\partial(a / 2)}\left[<\Psi_{2}\left|V_{1}\right| \Psi_{2}>-<\Psi_{1}\left|V_{1}\right| \Psi_{1}>\right]= \\
{\left[\frac{2 \Gamma \sqrt{2 / \pi}}{a\left(1-S^{2}\right)^{3 / 2}}\right]\left\{\Gamma^{2} a^{2} S^{2}\left[1-F_{0}\left(2 \Gamma^{2} a^{2}\right)\right]+\left(1-S^{2}\right)\left[S^{4}-F_{0}\left(2 \Gamma^{2} a^{2}\right)\right]\right\},}
\end{gathered}
$$

and

$$
\begin{gathered}
\frac{\partial}{\partial(a / 2)}\left[<\Psi_{2}\left|V_{1}\right| \Psi_{2}>+<\Psi_{1}\left|V_{1}\right| \Psi_{1}>\right]= \\
{\left[\frac{2 \Gamma \sqrt{2 / \pi}}{a\left(1-S^{2}\right)^{2}}\right]\left\{-2 \Gamma^{2} a^{2} S^{2}-(2-S) S^{3}\left(1-S^{2}\right)-F_{0}\left(2 \Gamma^{2} a^{2}\right)\left[1-S^{2}\left(1-2 \Gamma^{2} a^{2}\right)\right]\right.}
\end{gathered}
$$




$$
\left.+F_{0}\left(\frac{\Gamma^{2} a^{2}}{2}\right)\left[4 \Gamma^{2} a^{2} S^{2}+2 S^{2}\left(1-S^{2}\right)\right]\right\} .
$$

By substituting the equations above deduced and the other matrix elements into the Eq.(87) and Eq.(88) we obtain the unknown matrix elements as:

$$
\begin{gathered}
\left\langle\phi_{2}\left|\left(x-\frac{a}{2}\right) V_{1}\right| \phi_{2}\right\rangle=\frac{\sqrt{2 / \pi}}{2 a \Gamma}\left[S^{4}-F_{0}\left(2 a^{2} \Gamma^{2}\right)\right], \\
\left\langle\phi_{1}\left|x V_{1}\right| \phi_{2}\right\rangle=\frac{S \sqrt{2 / \pi}}{a \Gamma}\left[S-F_{0}\left(\frac{a^{2} \Gamma^{2}}{2}\right)\right] .
\end{gathered}
$$

\section{Appendix 4}

Let us evaluate the crystal field coupling for site 1 in the adiabatic limit, in order to verify Eq.43. For the charge on site 1 the variation of the local energy defines $g_{c f a d}^{(1)}$ as:

$$
\begin{gathered}
\int_{-\infty}^{\infty} \Psi_{1}\left(\boldsymbol{R}_{1}^{0}-\boldsymbol{u}_{1}, \boldsymbol{R}_{2}^{0}-\boldsymbol{u}_{2}, \boldsymbol{r}\right)^{2} V_{2}\left(\boldsymbol{R}_{2}^{0}-\boldsymbol{u}_{2}, \boldsymbol{r}\right) d^{3} \boldsymbol{r}-\int_{-\infty}^{\infty} \Psi_{1}\left(\boldsymbol{R}_{1}^{0}, \boldsymbol{R}_{2}^{0}, \boldsymbol{r}\right)^{2} V_{2}\left(\boldsymbol{R}_{2}^{0}, \boldsymbol{r}\right) d^{3} \boldsymbol{r} \\
\equiv g_{c f a d}^{(1)} n_{1}\left(u_{2}-u_{1}\right)+\mathcal{O}\left[\left(\sqcap_{\in}-\sqcap_{\infty}\right)^{\epsilon}\right] .
\end{gathered}
$$

Substituting $t=x-a / 2-u_{2}$ and developing $\Psi_{1}(u, t, y, z)^{2}$ to first order in $u$ yields:

$$
g_{\text {cfad }}^{(1)}=\left.2 \int_{-\infty}^{\infty} \Psi_{1}(0, t, y, z) \frac{\partial \Psi_{1}}{\partial(u)}\right|_{u=0} V_{2}(t, y, z) \bullet e_{12} .
$$

By substituting $t=x-a / 2-u_{2}$ we obtain:

$$
\begin{gathered}
\left.\frac{\partial \Psi_{1}}{\partial u}\right|_{u=0}=\left.\frac{\partial A}{\partial u}\right|_{u=0} N e^{-\Gamma^{2}\left[(t+a)^{2}+y^{2}+z^{2}\right]}+\left.\frac{\partial B}{\partial u}\right|_{u=0} N e^{-\Gamma^{2}\left[t^{2}+y^{2}+z^{2}\right]} \\
-2 \Gamma^{2} A(t+a) N e^{-\Gamma^{2}\left[(t+a)^{2}+y^{2}+z^{2}\right]}
\end{gathered}
$$

Substituting $\Psi_{1}$ and Eq.(99) into Eq.(98) yields:

$$
\begin{gathered}
g_{c f a d}^{(1)}=\left.\frac{\partial\left(A^{2}\right)}{\partial u}\right|_{u=0}\left\langle\phi_{1}\left|V_{2}\right| \phi_{1}\right\rangle+\left.\frac{\partial\left(B^{2}\right)}{\partial u}\right|_{u=0}\left\langle\phi_{2}\left|V_{2}\right| \phi_{2}\right\rangle+\left.2 \frac{\partial(A B)}{\partial u}\right|_{u=0}\left\langle\phi_{1}\left|V_{2}\right| \phi_{2}\right\rangle \\
+4 \Gamma^{2}\left[A_{0}^{2}\left\langle\phi_{1}\left|(x+a / 2) V_{2}\right| \phi_{1}\right\rangle+2 A_{0} B_{0}\left\langle\phi_{1}\left|x V_{2}\right| \phi_{2}\right\rangle\right] .
\end{gathered}
$$

The corresponding coupling for the other site is obtained by the site permutation $1 \rightleftharpoons 2$

$$
H_{c f a d}^{(2)}=g_{c f a d}^{(2)} \sum_{\sigma} n_{2 \sigma}\left(u_{1}-u_{2}\right)
$$

that is, with $t=x+a / 2-u_{1}$ :

$$
g_{c f a d}^{(2)}=\left.2 \int_{-\infty}^{\infty} \Psi_{2}(0, t, y, z) \frac{\partial \Psi_{2}}{\partial\left(u_{1}-u_{2}\right)}\right|_{0} V_{1}(t, y, z) .
$$


Performing the corresponding calculations we obtain:

$$
\begin{aligned}
g_{c f a d}^{(2)}=g_{c f a d}^{(1)}-4 \Gamma^{2}\{ & A B\left\langle\phi_{1}\left|x\left(V_{1}+V_{2}\right)\right| \phi_{2}\right\rangle+A^{2}\left(\left[\left\langle\phi_{1}\left|x V_{2}\right| \phi_{1}\right\rangle+\left\langle\phi_{2}\left|x V_{1}\right| \phi_{2}\right\rangle\right)\right] \\
& \left.+B^{2}\left(\left[\left\langle\phi_{2}\left|x V_{2}\right| \phi_{2}\right\rangle+\left\langle\phi_{1}\left|x V_{1}\right| \phi_{1}\right\rangle\right)\right]\right\}
\end{aligned}
$$

The difference between $g_{c f a d}^{(2)}$ and $g_{c f a d}^{(1)}$ vanishes because of the results reported in the Appendix 2, Eq.( 74 ).

\section{References}

1. J. R. Iglesias, M.A. Gusmão, M. Acquarone, A. Romano and C. Noce, Physica B 230232, 1047 (1997) and M. Acquarone, J. R. Iglesias, M.A. Gusmão, C. Noce and A. Romano, Phys. Rev. B 58, 7626 (1998).

2. S. Barisic, J.Labbé and J. Friedel, Phys. Rev. Letters 25,919 (1970).

3. S. Barisic, Phys. Rev.B5, 932 (1972).

4. We correct here a misprint in Ref.1. In Eq.(1) there, the term $V n_{1} n_{2}$ should read ( $V-$ $\left.J_{z} / 2\right) n_{1} n_{2}$. The parameter $V$ in the present work corresponds therefore to $V-J_{z} / 2$ there.

5. W.P. Su, J.R. Schrieffer and A.J. Heeger Phys. Rev.B22, 2099 (1980).

6. T. Holstein Annals of Physics 8, 325 (1959).

7. T. Sakai, D. Poilblanc and D.J. Scalapino Phys. Rev.B55, 8445 (1997).

8. S. Barisic Europhys. Lett. 8, 765 (1989); S. Barisic Int. J. Mod. Phys. 5, 2439 (1991).

9. A. L. Kuzemsky, A. Holas and N.M.Plakida, Physica 122B, 168 (1983).

10. L. Pietronero, S. Strassler and H. R. Zeller Phys. Rev. B22, 904 (1980).

11. A. A. Aligia, M. Kulic, V. Zlatic and K.H. Bennemann Sol. State Comm. 65, 501 (1988).

12. Z.K. Petru and N.M. Plakida, Modern Phys. Letters, B4, 489 (1990).

13. R. Hlubina and G.K.Sadiek Phys. Rev. B55, 2733 (1997).

14. B. K. Chakraverty, M. J. Sienko and J. Bonnerot Phys. Rev. B17, 3781 (1978).

15. A.N. Das and S. Sil J.Phys. Cond. Matter 5, 8265 (1993); A.N. Das and P. Choudhury Phys. Rev. B49, 13219 (1994).

16. A. Gołebiewski and J. Mrozek Int. J. Quantum Chem. 7, 623 and 1021,(1973).

17. E. Ya. Sherman, Sol. State Comm. 104, 619 (1997). 